\title{
Eventos reproductivos del león marino común, Otaria flavescens (Shaw 1800), en el norte de Chile (Pacífico suroriental)
}

\author{
Reproductive events of southern sea lion, Otaria flavescens (Shaw 1800), \\ in the north of Chile (eastern south Pacific)
${ }^{1}$ Centro de Estudios del Cuaternario (CEQUA). Avda. Bulnes 01855, Punta Arenas, Chile.
${ }^{2}$ Programa de Mamíferos Marinos, Departamento Científico del Instituto Antártico Chileno, Plaza Muñoz Gamero 1055, Punta Arenas, Chile.
${ }^{3}$ Departamento de Ciencias del Mar, Universidad Arturo Prat. Casilla 121, Iquique, Chile. jacevedo@123mail.cl \\ Jorge Acevedo R. ${ }^{1,2}$, Anelio Aguayo-Lobo ${ }^{1,2}$ y Walter Sielfeld K. ${ }^{3}$
}

\begin{abstract}
Resumen.- Estudios sobre el desarrollo de la temporada de reproducción del león marino común han sido largamente realizados en poblaciones de Uruguay y Argentina, Atlántico suroriental, disponiéndose de escasa información en Perú y Chile. El objetivo de esta contribución es dar a conocer el desarrollo de la temporada de reproducción del león marino del sur, durante dos períodos consecutivos en la lobera de Punta Negra, norte de Chile.

El desarrollo de los eventos reproductivos en la lobera de Punta Negra presentó una sincronización anual, extendiéndose desde la tercera semana de diciembre hasta mayo, con un clímax de los eventos reproductivos en febrero, siendo la dinámica del período de reproducción similar a la informada para otras colonias estudiadas, aunque con un desfase en el tiempo en contraste con las poblaciones de la costa atlántica.

Se concluye que el desarrollo de los eventos durante ambas temporadas de reproducción presentó una alta sincronización anual, con un desfase promedio de 2,0 - 2,5 días, pudiendo ser generalizada como representativa para la zona norte de Chile, en el Pacífico suroriental.
\end{abstract}

Palabras claves: Lobo marino común, Otaria flavescens, temporada de reproducción, Chile

\begin{abstract}
Studies on the development of the breeding season of southern sea lions have been carried out primarily in populations from Uruguay and Argentina, western south Atlantic; scarce information has been published from populations of Peru and Chile. The objective of this contribution is to describe the development of the breeding season of the southern sea lion, during two consecutive seasons in the area of Punta Negra, north of Chile.

The development of the reproductive events in the Punta Negra colony presented a high annual synchronization, extending from the third week of December till May, with a climax of the reproductive events in February, being the dynamics during the breeding period similar to that informed for other colonies, although with an out of fase in the breeding season with the Atlantic populations of sea lion.

We conclude that the development of the reproductive events during the both breeding seasons presented a high annual synchronization, with an average out of fase of 2.0-2.5 days, being representative for most of the northern area of Chile, in the southeastern Pacific Ocean.
\end{abstract}

Key words: Southern sea lion, Otaria flavescens, breeding season, Chile

\section{Introducción}

El lobo marino común es relativamente abundante a lo largo del litoral Pacífico, desde bahía Zorritos (Perú) (Scheffer 1958) hasta las islas Diego Ramírez (Chile) (Pisano 1972; Schlatter \& Riveros 1997), no habiendo evidencias confiables para el archipiélago de Juan Fernández (Aguayo \& Maturana 1973). Felix et al. (1994) informan de diez registros confirmados de machos adultos y subadultos en las costas de Ecuador entre los años 1989 a 1994, registros que para Acevedo (1999) extenderían la distribución hasta los $00^{\circ} 35^{`} \mathrm{~N}$. En la costa atlántica, se distribuye desde Río de Janeiro (Brasil) hasta Tierra del Fuego e islas Malvinas o
Falkland e islas de los Estados (Osgood 1943; Vaz Ferreira 1981; King 1983; Bonner 1994; Rice 1998).

El estado sobre el conocimiento de esta especie en Chile es en general incompleto e insuficiente. Ello es especialmente válido en lo concerniente a la biología reproductiva y dinámica poblacional, donde los esfuerzos de estudios de los últimos años se han centrado fundamentalmente en estimaciones del tamaño poblacional (Sielfeld 1999).

El desarrollo de la temporada de reproducción del león marino común, Otaria flavescens (Shaw 1800), ha sido largamente estudiado en poblaciones de la costa atlántica de América del Sur, destacando aquellos de las 
islas Malvinas o Falkland (Hamilton 1934, 1939), isla Lobos, Uruguay (Vaz Ferreira 1975, 1981, 1982) y península Valdés y zonas adyacentes de la Provincia de Chubút, Argentina (Ximénez 1975; Campagna 1985). Contrariamente, en la costa pacífica de Sudamérica, se dispone de escasa información sobre la biología de la reproducción, en especial para Chile, a excepción de dos trabajos de tesis realizados en la Universidad Arturo Prat, Iquique, Chile (Araya et al. 1986, Acevedo 1999).

El objetivo de esta contribución es dar a conocer el desarrollo de la temporada de reproducción del león marino del sur, durante dos temporadas consecutivas en la zona norte del país.

\section{Materiales y Métodos}

El estudio se realizó en la lobera de Punta Negra (2050'S; 70¹0`W) localizado a $80 \mathrm{~km}$ al sur de la ciudad de Iquique, I Región de Chile (Fig. 1). La lobera está constituida por un islote rocoso principal de $90 \mathrm{~m}$ de longitud y $45 \mathrm{~m}$ de anchura máxima, estimándose una superficie de $1.800 \mathrm{~m}^{2} \mathrm{y}$, separado de la costa por un estrecho canal de aproximadamente $15 \mathrm{~m}$ de anchura.

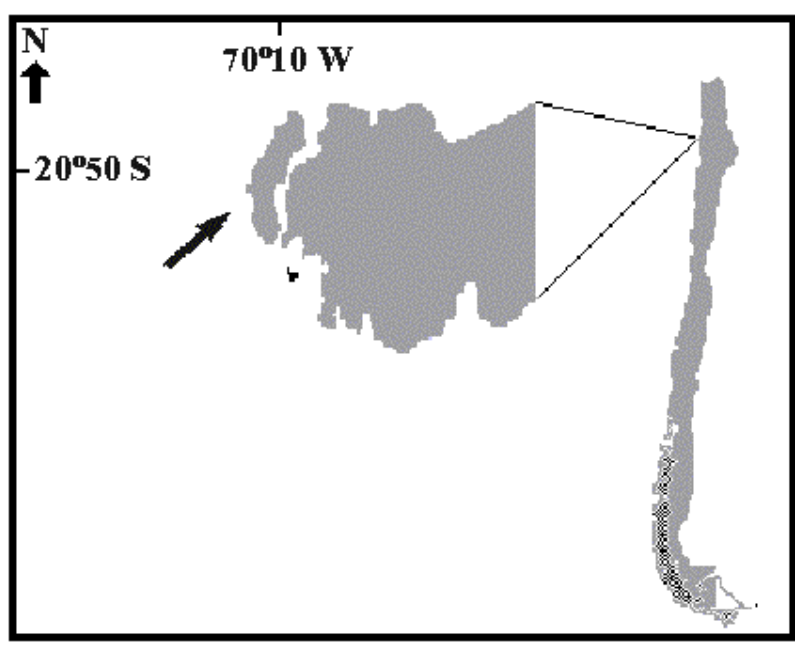

Figura 1

Área de estudio y localización de la lobera de Punta Negra (flecha), Iquique, norte de Chile

Map of the main islet where the breeding season is developed at Punta Negra (arrow), Iquique, north of Chile
Censos periódicos y dobles de lobos marinos comunes se efectuaron en la mañana (09:00 a 11:00) y tarde (17:00 a 19:00) entre diciembre e inicios de marzo de la temporada 1995/1996 y, entre diciembre y mayo de la temporada 1996/1997. Los conteos fueron realizados desde una torre de observación de $6 \mathrm{~m}$ de altura y a una distancia aproximada de $35 \mathrm{~m}$ de la lobera usando binoculares $8 \times 40$ y un telescopio Tasco 21EB montado sobre trípode. Se consideraron los valores censales máximos como representativos de cada día.

Los animales se clasificaron en machos adultos, machos subadultos, hembras adultas, juveniles y cachorros, según parámetros morfológicos externos observables a simple vista (Aguayo-Lobo et al. 1998). La categoría de machos adultos fue dividida en dos subcategorías de acuerdo a su distribución en la lobera, considerándose como macho territorial sólo a aquellos ejemplares que presentaban hembras en sus territorios.

En el desarrollo de la temporada de reproducción se consideraron como eventos importantes a destacar, las fechas de inicio, máximo de llegada y abandono de los machos reproductores y hembras adultas, como así también las fechas de inicio, máximo y término de los partos y cópulas. Las primeras fueron determinadas a través de censos periódicos, mientras que las fechas de inicio y término de los partos y de las cópulas, fueron determinadas a través de registros de observaciones directas efectuadas durante 12 horas diarias de permanencia en cada censo.

Las fechas de máxima frecuencia de partos se estimaron mediante un histograma confeccionado sobre la base de las diferencias en la cantidad de crías censadas presentes en la lobera entre censos consecutivos; mientras que la máxima frecuencia de cópulas, se obtuvo a través de un histograma de los registros de cópulas observados durante los censos.

\section{Resultados y Discusión}

En la Fig. 2 se presentan los censos de machos adultos territoriales, hembras adultas y cachorros de ambas temporadas de reproducción, como un promedio por períodos de siete días desde diciembre hasta marzo. Se ha excluido de la figura los censos correspondientes a machos subadultos y juveniles, por la alta variabilidad mostrada entre ambas temporadas. La Fig. 2 muestra que la presencia de machos territoriales, hembras adultas y cachorros desde diciembre hasta marzo presentó un desarrollo similar durante ambas temporadas de reproducción, la cual estaría sugiriendo 
la existencia de una posible sincronización anual en el desarrollo de los eventos reproductivos.

Al analizar el desarrollo de los eventos más destacados en ambas temporadas de reproducción, (Tabla 1) se observa que las fechas durante las temporadas de 1995/1996 y 1996/1997 fueron muy similares entre sí (desfase: 0-6 días), estimándose un desfase promedio, al considerar las fechas medias, de tan sólo 2,5 $\pm 2,07$ días ( $n=8$ eventos). Este pequeño desfase obtenido entre ambas temporadas de reproducción estaría indicando la existencia de una sincronización anual en el desarrollo de los eventos reproductivos $\mathrm{y}$, por tanto, de la temporada de reproducción en la lobera de Punta Negra. Es así que, se puede observar que tanto el arribo de los primeros machos adultos territoriales y hembras adultas, así como las fechas en que se alcanza el máximo de llegada de ambas categorías etarias, presenta una alta sincronía, con un desfase de tan sólo 0 a 2 días.
Al comparar estos mismos eventos reproductivos de las temporadas 1995/1996 y 1996/1997 con la información presentada por Araya et al. (1986) para la misma lobera durante 1984/1985, se observa que las fechas de desarrollo de los eventos fueron muy similares entre sí (variación: 0-6 días), estimándose un desfase promedio de tan sólo 2,0 $\pm 1,87$ días ( $\mathrm{n}=9$ eventos) (Tabla 2).

Esta baja variabilidad en las fechas en que ocurrieron estos eventos reproductivos, estaría indicando que el desarrollo anual de la temporada de reproducción es altamente sincronizada en la lobera de Punta Negra, hecho que podría ser generalizada como representativa, al menos, para la zona norte de Chile. Cabe destacar que las tres temporadas tratadas comparativamente, correspondieron a años sin fenómenos "El Niño", desconociéndose si esta sincronización anual es afectada en presencia de dicho fenómeno oceanográfico.

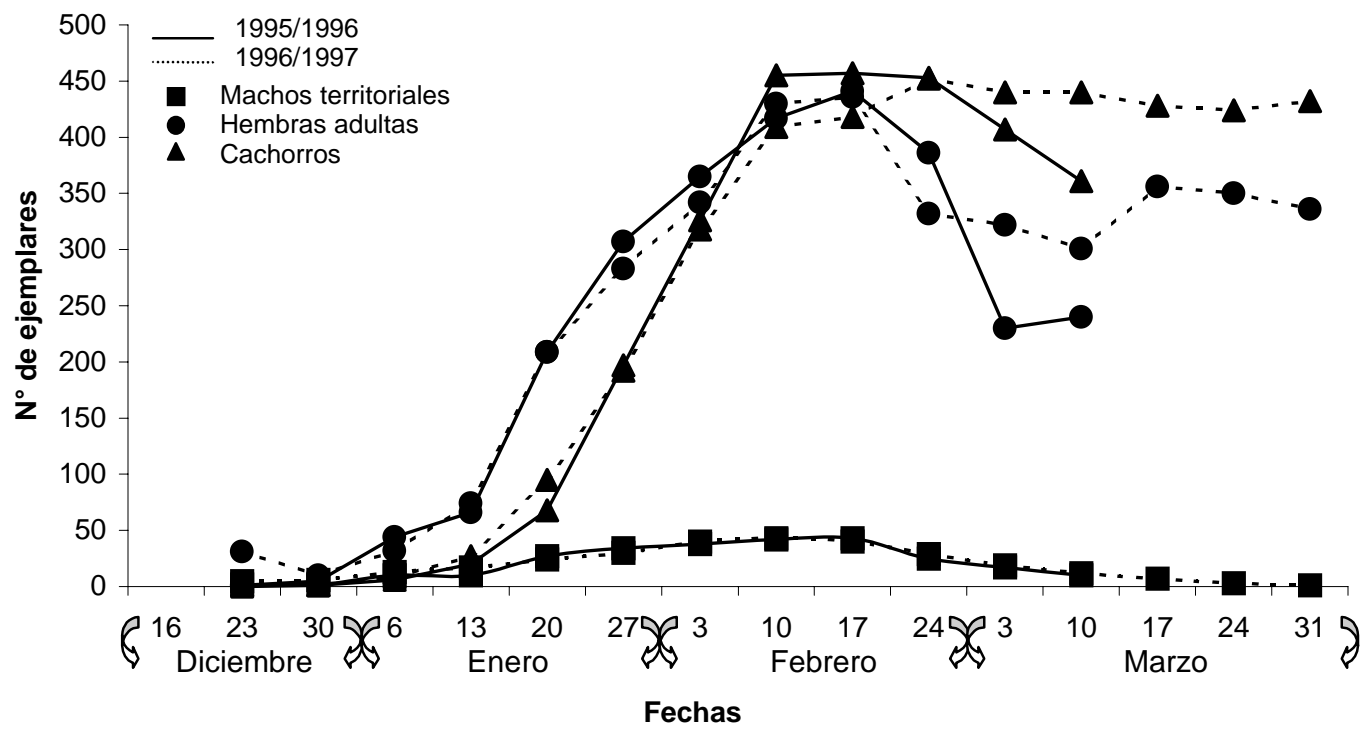

Figura 2

Número promedio de machos adultos territoriales, hembras adultas y cachorros censados durante las temporadas de reproducción 1995/1996 y 1996/1997 en la lobera de Punta Negra, I Región,

Chile. Los censos fueron agrupados en períodos de siete días para su mejor ilustración

Average number of bulls, adult females and pups population, during the 1995/1996 and 1996/1997 breeding seasons in Punta Negra. I Region, Chile. The censuses were grouped in

7-days periods for better understanding 


\section{Tabla 1}

Fechas de ocurrencia de los eventos durante la temporada de reproducción del lobo común en la lobera de Punta Negra, I Región de Chile, en 1995/1996 y 1996/1997. (sr) sin registro

Temporary ocurrence of the reproductive events during the breeding season of the sea lion at Punta Negra, north of Chile, in 1995/1996 and 1996/1997. (sr) without record

\begin{tabular}{|c|c|c|c|c|c|}
\hline Eventos & 1995/1996* & $\begin{array}{l}\text { Fecha } \\
\text { media }\end{array}$ & $1996 / 1997$ & $\begin{array}{l}\text { Fecha } \\
\text { media }\end{array}$ & Desfase \\
\hline $1^{\mathrm{er}}$ macho territorial registrado & 23 Dic & 23 Dic & 21 Dic & 21 Dic & 02 días \\
\hline Máximo de machos territoriales & 11 Feb-15 Feb & $13 \mathrm{Feb}$ & 09 Feb-15 Feb & $13 \mathrm{Feb}$ & 00 días \\
\hline Abandono último macho territorial & $\mathrm{sr}$ & $\mathrm{sr}$ & $28 \mathrm{Mar}$ & $28 \mathrm{Mar}$ & $\mathrm{sr}$ \\
\hline $1^{\mathrm{a}}$ hembra registrada & 23 Dic & 23 Dic & $21 \mathrm{Dic}$ & $21 \mathrm{Dic}$ & 02 días \\
\hline Máximo de hembras & $15 \mathrm{Feb}$ & $15 \mathrm{Feb}$ & $14 \mathrm{Feb}$ & $14 \mathrm{Feb}$ & 01 días \\
\hline Abandono última hembra & $\mathrm{sr}$ & $\mathrm{sr}$ & > 18 May & > 18 May & $\mathrm{sr}$ \\
\hline $1^{\mathrm{er}}$ parto & 27 Dic & 27 Dic & 22 Dic & 22 Dic & 05 días \\
\hline Máxima frecuencia de partos & 30 Ene-05 Feb & $02 \mathrm{Feb}$ & 31 Ene-05 Feb & $03 \mathrm{Feb}$ & 01 días \\
\hline Ultimo parto & $\mathrm{sr}$ & $\mathrm{sr}$ & $26 \mathrm{Feb}$ & $26 \mathrm{Feb}$ & sr \\
\hline $1^{\mathrm{a}}$ cópula observada & 10 Ene & 10 Ene & 04 Ene & 04 Ene & 06 días \\
\hline Máxima frecuencia de cópulas & 28 Ene-11 Feb & $04 \mathrm{Feb}$ & 25 Ene-20 Feb & 07 Feb & 03 días \\
\hline Ultima cópula observada & sr & sr & $02 \mathrm{Mar}$ & $02 \mathrm{Mar}$ & sr \\
\hline
\end{tabular}

*Fecha última observación en terreno: 06 de marzo 1996

Tabla 2

Comparación de las fechas de ocurrencia de los eventos reproductivos del lobo común en la lobera de Punta Negra, I Región de Chile, entre las temporadas 1984/1985 (Araya et al. 1986) y 1995/1996-1996/1997 (este trabajo). (rd) registro dudoso; (sr) sin registro

Comparison of the date of the reproductive events in the southern sea lion at Punta Negra colony, Chile, between 1984/1985 season (Araya et al. 1986) and 1995/1996-1996/1997 (this work).

(rd) dubious record; (sr) without record

\begin{tabular}{|c|c|c|c|c|c|}
\hline Eventos & $1984 / 1985$ & $\begin{array}{l}\text { Fecha } \\
\text { media }\end{array}$ & $\begin{array}{l}1995-1996 \\
1996-1997 \\
\end{array}$ & $\begin{array}{l}\text { Fecha } \\
\text { media }\end{array}$ & Desfase \\
\hline $1^{\mathrm{er}}$ macho territorial registrado & 22 Dic & 22 Dic & 21 Dic-23 Dic & 22 Dic & 00 días \\
\hline Máximo de machos territoriales & 05 Feb-19 Feb & 13 Feb & 09 Feb-15 Feb & $12 \mathrm{Feb}$ & 01 días \\
\hline Abandono último macho territorial & 03 May* & rd & $28 \mathrm{Mar}$ & $28 \mathrm{Mar}$ & sr \\
\hline $1^{\mathrm{a}}$ hembra registrada & 28 Dic & $28 \mathrm{Dic}$ & 21 Dic-23 Dic & 22 Dic & 06 días \\
\hline Máximo de hembras & 16 Feb-17 Feb & 17 Feb & 14 Feb-15 Feb & $15 \mathrm{Feb}$ & 02 días \\
\hline Abandono última hembra & $>18$ May & $\begin{array}{l}>18 \\
\text { May }\end{array}$ & $>18$ May & > 18 May & 00 días \\
\hline $1^{\mathrm{er}}$ parto & 28 Dic & 28 Dic & 22 Dic-27 Dic & 25 Dic & 03 días \\
\hline Máxima frecuencia de partos & 27 Ene-09 Feb & 03 Feb & 30 Ene-05 Feb & $02 \mathrm{Feb}$ & 01 días \\
\hline Ultimo parto & $\mathrm{Sr}$ & sr & 26 Feb & $26 \mathrm{Feb}$ & $\mathrm{sr}$ \\
\hline $1^{\text {a }}$ cópula observada & 10 Ene & 10 Ene & 04 Ene-10 Ene & 07 Ene & 03 días \\
\hline Máxima frecuencia de cópulas & 01 Feb-09 Feb & 05 Feb & 25 Ene-20 Feb & $07 \mathrm{Feb}$ & 02 días \\
\hline Ultima cópula observada & 11 Mar* & rd & $02 \mathrm{Mar}$ & $02 \mathrm{Mar}$ & $\mathrm{sr}$ \\
\hline
\end{tabular}

*No se tiene certeza del registro 
Esta sincronización en la ocurrencia de los eventos reproductivos ha sido informada por Campagna (1985) al comparar una data de 5 años de las fechas de los principales eventos reproductivos de la población de lobos comunes en la lobera de Punta Norte $\left(42^{\circ} 04^{\prime} \mathrm{S}\right)$, Península Valdés, Argentina. Más aún, esta sincronización de los eventos reproductivos también ha sido informada para otras especies de Pinnipedia. Así por ejemplo, Payne (1977) señala para Arctocephalus gazella (Peters 1875) que las fechas de los principales eventos, tal como la máxima concentración de nacimientos, no varían por más de unos días, año tras año, en las islas Georgia del Sur.

Por su parte, la dinámica durante la temporada de reproducción se inicia con el arribo de los primeros machos adultos y hembras durante la tercera semana de diciembre, siendo notorio el arribo de ambas categorías etarias desde los primeros días de enero hasta mediados de febrero. Durante este período, los machos compiten agresivamente en el establecimiento y mantención de su posición en la lobera, residiendo en densas agregaciones (territorios o harén) con acceso a las pozas de agua. Así mismo, al arribar las hembras preñadas a la lobera, estas se integran a los distintos territorios, en especial en aquellos territorios que poseen pozas de aguas. Aunque los primeros nacimientos en ambas temporadas se registraron durante la segunda quincena de diciembre (22 y 27 de diciembre) ninguno de estos cachorros sobrevivió al día siguiente y ningún parto múltiple fue registrado. No obstante, el nacimiento de crías viables fue registrado desde inicios de enero.

Entre mediados de enero y fines de febrero, la temporada de reproducción alcanza su clímax. Los machos territoriales se muestran muy activos y compiten agresivamente en la mantención de su territorio y de las hembras adultas (5 a 20 hembras adultas por macho territorial), estimándose una proporción sexual promedio de 1:12 para ambas temporadas de reproducción. Así mismo, cambios numéricamente distinguibles en la cantidad de hembras adultas se registraron entre las horas matutinas y vespertinas desde mediados de febrero, siendo mayor la presencia de hembras adultas en la lobera durante este último horario.

No obstante de que el nacimiento de crías viables se registra desde inicios de enero, la máxima frecuencia de nacimientos fue registrada entre el 30 - 31 de enero y 05 de febrero (Tabla 1), siendo el inicio de la máxima frecuencia de nacimientos coincidente con el 50\% del total de cachorros nacidos y, el término, con el 75\% del total de las crías nacidas. El parto presentó una duración promedio de $16 \pm 7,2$ minutos (rango: $6-34$ min; $n=$ 176), y en una proporción de 1 nacimiento cefálica por cada 3,5 caudales. Por su parte, la totalidad de las cópulas exitosas observadas en ambas temporadas ( $\mathrm{n}=$ 81) tuvo lugar en tierra con una duración promedio de 8,69 \pm 2,94 minutos (rango: $5-17 \mathrm{~min}$ ) y fueron llevadas a cabo por machos territoriales, con excepción de dos casos realizados por machos subadultos, ubicados en la periferia de la lobera.

A fines de febrero, la actividad de reproducción comienza a declinar, disolviéndose lentamente la estructura reproductiva con el abandono paulatino de los machos territoriales, y posteriormente de las hembras adultas con sus cachorros durante mayo.

La presencia de machos adultos periféricos (no territoriales), subadultos y juveniles fue altamente variable entre ambas temporadas de reproducción e incluso en el lapso de un mismo día, mostrando además una distribución diferencial. Los machos adultos periféricos se distribuyeron en plataformas rocosas adyacentes a la lobera principal. Los machos subadultos se distribuyeron tanto en la periferia de la lobera como en islotes adyacentes a ella; mientras que los juveniles se distribuyeron en lugares periféricos del centro principal de actividad.

La dinámica de la temporada de reproducción descrita para la lobera de Punta Negra, presentó el mismo desarrollo informado por Araya et al. (1986) para la misma localidad, como también, para otras poblaciones de esta especie (Hamilton 1934, 1939; Vaz Ferreira 1965, 1981, 1982; Ximénez 1975; Campagna 1985). No obstante, cabe destacar que el desarrollo de la temporada de reproducción descrita en este trabajo ocurre más tardíamente que en las poblaciones de la costa atlántica, en donde el clímax de la temporada se alcanza en enero (Acevedo et al. 2001'; Acevedo \& Aguayo-Lobo 2002²).
1 Acevedo, J, A Aguayo-Lobo \& W Sielfeld. 2001. La reproducción del león marino del sur, Otaria flavescens, en el norte de Chile (Pacífico suroriental) y su comparación con el de isla Lobos, Uruguay (Atlántico suroccidental). XXI Congreso de Ciencias del Mar. Facultad de Ciencias del Mar, Universidad de Valparaíso, Chile. Viña del Mar, 22 al 25 de mayo de 2001. Resumen, p. 3.

2 Acevedo, J \& A Aguayo-Lobo. 2002. Comparación de la ocurrencia temporal de los eventos reproductivos del león marinos del sur, Otaria flavescens (Shaw 1800), entre la población del norte de Chile (Pacífico suroriental) y del Atlántico suroccidental. 10 Reunión de Trabajo de Especialistas en Mamíferos Acuáticos de América del Sur y $4^{\circ}$ Congreso de la Sociedad Latinoamericana de Especialistas de Mamíferos Acuáticos. Valdivia, 14 al 19 de octubre de 2002. Resumen, p. 25. 


\section{Tabla 3}

Ocurrencia de los principales eventos reproductivos desarrollados durante las temporadas de 1995/1996 y 1996/1997, en la lobera de Punta Negra, I Región, Chile

Ocurrence of the main reproductive events at Punta Negra, north of Chile, during the 1995/1996 and 1996/1997 reproductive seasons

\begin{tabular}{|c|c|c|c|c|c|c|}
\hline Actividad / mes & Diciembre & Enero & Febrero & Marzo & Abril & Mayo \\
\hline Inicio arribos de machos territoriales & $21-23$ & & & & & \\
\hline Inicio arribos de hembras adultas & $21-23$ & & & & & \\
\hline $\mathrm{N}^{\mathrm{o}}$ máximo de machos territoriales & & & $5-19$ & & & \\
\hline $\mathrm{N}^{\mathrm{o}}$ máximo de hembras adultas & & & $14-17$ & & & \\
\hline $\mathrm{N}^{\mathrm{o}}$ máximo de cachorros & & & 26 & & & \\
\hline Inicio desorganización lobera & & & & $01-03$ & & \\
\hline Abandono del último macho territorial & & & & 28 & & \\
\hline Abandono de hembras adultas y crías & & & & & & $>18$ \\
\hline Primer parto & $22-28$ & & & & & \\
\hline Máxima frecuencia de partos & & \multicolumn{2}{|c|}{$27-09$} & & & \\
\hline Último parto & & & 26 & & & \\
\hline Inicio de cópulas & & $04-10$ & & & & \\
\hline Máxima frecuencia de cópulas & & & & & & \\
\hline Última cópula & & & & 02 & & \\
\hline
\end{tabular}

De acuerdo a lo expuesto, en la Tabla 3 se presenta a modo ilustrativo y en orden de secuencia cronológica, el desarrollo de los eventos reproductivos durante la temporada de reproducción para la zona norte de Chile.

\section{Conclusiones}

1. El desarrollo de la temporada de reproducción en la lobera de Punta Negra se extiende desde la tercera semana de diciembre hasta mayo, alcanzándose el clímax de la temporada en febrero.

2. El desarrollo de los eventos durante la temporada de reproducción presenta una alta sincronización anual, con un desfase promedio de 2,0 - 2,5 días, pudiendo ser generalizada como representativa para la zona norte de Chile.

3. El desarrollo de la dinámica durante la temporada de reproducción es coincidente con lo informado para otras localidades reproductivas de esta misma especie, aunque con un desfase en el tiempo en contraste a las poblaciones de la costa atlántica.

\section{Agradecimientos}

Se agradece al Centro de Estudios del Cuaternario Fuego-Patagonia y Antártica (CEQUA) y al Instituto Antártico Chileno (INACH) por todas las facilidades administrativas y de espacio físico otorgados a los integrantes del Grupo de Biología Marina. Además, se agradece a los correctores anónimos por sus valiosas sugerencias realizadas al manuscrito.

\section{Literatura citada}

Acevedo J. 1999. Temporada de reproducción del lobo marino común, Otaria flavescens (Shaw, 1800), en la lobera de Punta Negra (2050`S), 1995/96 y 1996/97: Crías. Tesis para optar al Título de Biólogo Marino. Universidad Arturo Prat, Iquique, Chile. 150 págs +6 anexos.

Aguayo A \& R Maturana. 1973. Presencia del lobo marino común Otaria flavescens en el litoral chileno. I. Arica $\left(18^{\circ} 20^{\prime} \mathrm{S}\right)$ a Punta Maiquillahue $\left(39^{\circ} 27^{\prime} \mathrm{S}\right)$. Biología Pesquera 6: $45-75$. 
Aguayo-Lobo A, H Díaz, J Yánez, F Palma \& M Sepúlveda. 1998. Censo poblacional del lobo marino común en el litoral de la $\mathrm{V}$ a la IX Regiones. Informe Final Proyecto FIP 96-51. Doppler Ltda. Valparaíso. 214 págs +2 anexos.

Araya H, M Arroyo, F Campos \& F Contreras. 1986. Conducta reproductiva del lobo marino común (Otaria flavescens) en Punta Negra, Iquique - Chile. Tesis para optar al Título de Profesor de Estado en Biología y Ciencias. Universidad Arturo Prat, Iquique, Chile. 165 pp.

Bonner N. 1994. Seals and sea lions of the world. Blandford, U.K., 224 pp.

Campagna C. 1985. The breeding cycle of the Southern sea lion, Otaria byronia. Marine Mammals Science 1 (3): 210-218.

Félix F, J Samaniego \& J Oechsle. 1994. New evidence of the presence of the South American Sea Lions Otaria flavescens (Carnivora: Pinnipedia) in Ecuatorian waters. Estudios Oceanológicos 13: 85-88.

Hamilton J. 1934. The Southern sea lion Otaria byronia (de Blainville). Discovery Reports 8: 269-318.

Hamilton J. 1939. A second report on the Southern sea lion Otaria byronia (de Blainville). Discovery Reports 19: 121-164.

King J. 1983. Seals of the world. Oxford University Press. Cambridge, U.K., 240 pp.

Osgood W. 1943. The Mammals of Chile. Publication Field Museum of Natural History (Zoology) Series. 30. Chicago, 268 pp.

Payne M. 1977. Growth of a fur seal population. Philosophical Transaction of the Royal Society of London 279: 67-79.
Pisano E. 1972. Observaciones fito-ecológicas en las islas Diego Ramírez. Anales del Instituto de la Patagonia (Chile) 3 (1-2): 161-169.

Rice DW. 1998. Marine Mammals of the World. Systematic and Distribution. Special Publication Number 4. The Society for Marine Mammalogy, 231 pp.

Schlatter RP \& GM Riveros. 1997. Historia natural del archipiélago Diego Ramírez, Chile. Serie Científica INACH 47: 87-112.

Scheffer VB. 1958. Seals, Sea Lions and Walruses. A review of the Pinnipedia. Stanford University Press, 179 pp.

Sielfeld W. 1999. Estado del conocimiento sobre conservación y preservación de Otaria flavescens (Shaw, 1800) y Arctocephalus australis (Zimmermann, 1783) en las costas de Chile. Estudios Oceanológicos 18: 81-96.

Vaz Ferreira R. 1975. Behavior of the Southern sea lion Otaria flavescens in the Uruguayan Islands. Rapports et Procès-Verbaux des Rèunions. Council International Pour L’Exploration de la Mer. 169: 219-227.

Vaz Ferreira R. 1981. South American sea lion. En: Ridgway SH \& RJ Harrison (eds), Handbook of Marine Mammals, pp. 39-65. Academic Press.

Vaz Ferreira R. 1982. Otaria flavescens (Shaw), south american sea lion. FAO Fisheries Series IV (5): 477 - 494.

Ximénez I. 1975. Dinámica de la población de Otaria flavescens (Shaw) en el área de Península Valdés y zonas adyacentes (Provincia del Chubut, República Argentina). Comisión Nacional de Estudios Geo-Heliofísicos, Centro Nacional Patagónico. C.N.P. - 1.4-1, 52 pp. 